\title{
Experimental investigation of Ethane and Propane injection under sub- and super-critical conditions
}

\author{
N. Vallée, G. Ribert*, J.-B. Blaisot, D. Lisiecki \\ Normandie Univ., INSA Rouen Normandie, UNIROUEN, CNRS, CORIA \\ 76000 Rouen, France \\ *Corresponding author: ribert@coria.fr
}

\begin{abstract}
Studying a fluid flow under high-pressure conditions through reliable experiments is still nowadays a challenge. When the chamber pressure exceeds the critical pressure of working fluids the supercritical state of matter is reached and the distinction between gas and liquid becomes blurred. For such special conditions, experimental data are scarce and need to be consolidated. Indeed, the modification of the local refractive index induced by density gradient needs to be analyzed with appropriate image-based technique.

In the present study, the REFINE test bench (Real-gas Effect on Fluid Injection: a Numerical and Experimental study) has been designed at CORIA Lab to study the non-reactive injection of Ethane and Propane into Nitrogen under sub- and supercritical conditions. The ambient gas pressure can be raised up to $6 \mathrm{MPa}$ and warmed up to $573 \mathrm{~K}$ to scan sub- and trans-critical injections. The chamber is equipped with two perpendicular optical axes allowing simultaneously different optical diagnostics. Experimental data are collected from shadowgraph and diffused backlight illumination techniques. Quantitative measurements of jet spreading angle and breakup length are compared to results coming from literature exhibiting multiple flows topologies.
\end{abstract}

\section{Keywords}

Supercritical, Experimental, Injection.

\section{Introduction}

In propulsion applications like in rocket engines, gas turbines or diesel engines when late injections are performed, injection often occurs at pressures that are above the critical point of the incoming fluids, i.e. at supercritical pressures [1, 2]. At such high-pressure conditions, injection is quite different from classical known behaviors observed at low pressure, and studying these new phenomena is challenging. Very few experiments dealing with non-reacting supercritical injection exists in the world, but a substantial experimental data set has been developed over the last few decades at DLR and AFRL laboratories [3]. The main issue is the ambient high pressure that locally affects the refractive index gradient making the delivery of experimental data highly challenging. Therefore this lack of reliable data is damaging for the development of models for turbulence and combustion $[4,5,6]$.

The objectives of the research program REFINE (Real-gas Effects on Fluid Injection: a Numerical and Experimental study) are to bridge the gap between experimental and numerical observations in providing reliable experimental data and new strategies of modeling. The experimental aspect is presently addressed through the development of a new test-bench (next section) and for which a first qualitative analysis is presented. Indeed, many combustion devices involve thermodynamic phase transition from subcritical to supercritical state as in high-pressure combustion chambers of rocket or diesel engines. When the pressure exceeds the critical pressure $p_{c}$ of any substance, the distinction between gas and liquid vanishes and the injection and combustion are quite different from classical known behaviors observed at low pressure. $p_{c}$ (resp. $T_{c}$ ) is the highest pressure (resp. temperature) for which distinct liquid and gas phases can be observed [7]. Above these values spray atomization is changed [3, 8, 9]: drops are no longer observed on Shadowgraph or Schlieren images due to the reduced surface tension and heat of vaporization [10,11]. Nevertheless a dense and dark core is still visible at the injector exit on shadowgraph [12]. The analysis of the spreading angle shows that supercritical jet has a behavior closer to a gaseous jet than to a liquid one [13, 14]. Only a few studies are dealing with experimental investigations of high-pressure injection and mixing process. Moreover, existing test benches often include additional effects that add a degree of complexity to a process already complex in nature. Indeed, particular conditions such as reactive flows, assisted-jet, cryogenic injection or acoustic interactions are often considered.

This paper introduces a new test bench and brings the first quantitative results that are compared to the literature. Injection of Propane was first studied but more investigations were explored for Ethane jets with in particular, the influence of the injection velocity, the temperature of the surrounding fluid, and the pressure. A first set of data on breakup length and spreading angle is also provided.

\section{Experimental setup}

The experiment has been designed to study injection and atomization of a non-assisted jet under high pressures conditions. REFINE is equipped with a steel chamber able to withstand pressures up to $10 \mathrm{MPa}$ and moderate temperatures $(573 \mathrm{~K})$. Two optical axes through circular silica windows of $\varnothing 120 \mathrm{~mm}$ are available for simultaneous measurements (see Fig. 1). The chosen working fluids are Ethane $\left(\mathrm{C}_{2} \mathrm{H}_{6}, T_{c}=305.3 \mathrm{~K}\right.$ and $\left.p_{c}=4.87 \mathrm{MPa}\right)$ and Propane $\left(\mathrm{C}_{3} \mathrm{H}_{8}, T_{c}=369.8 \mathrm{~K}\right.$ and $\left.p_{c}=4.25 \mathrm{MPa}\right)$. Their moderate critical pressure and temperature are within 
the range of operating conditions achievable in the chamber. A one-liter pump supplies the working fluid at the liquid-gas equilibrium pressure. Before injection, the pump is filled up, and then the fluid contained inside the vessel of the pump is compressed to the target pressure plus $0.05 \mathrm{MPa}$ to prevent the ambient fluid in the main chamber from entering the pump and creating an effervescent jet.

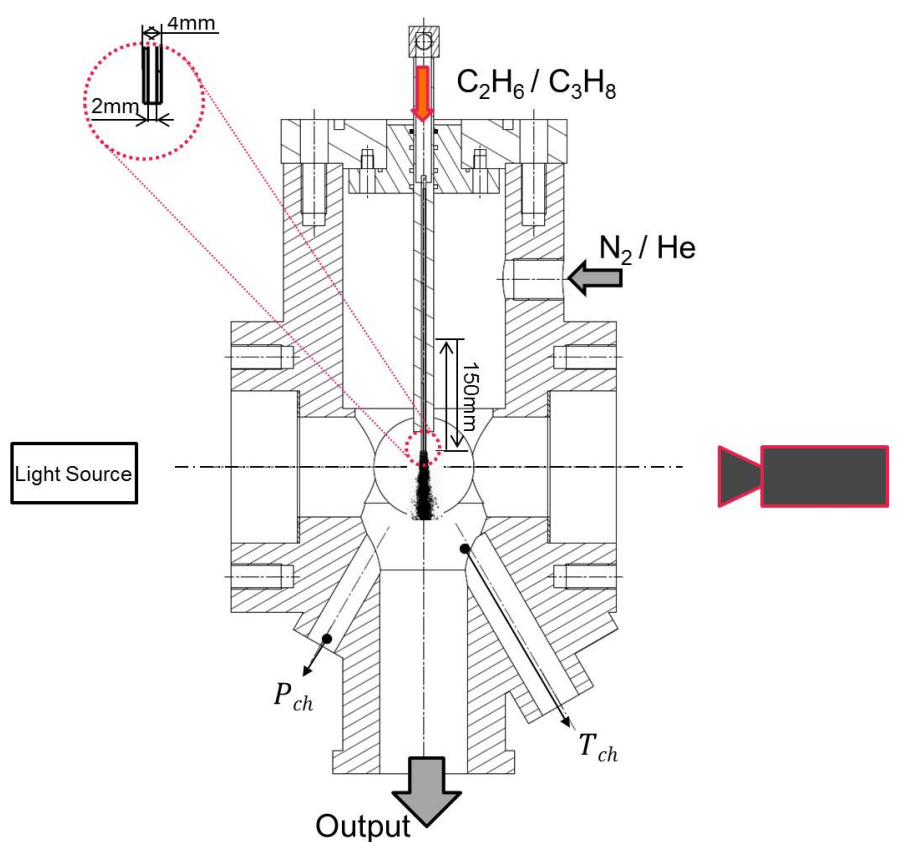

Figure 1. Section of the REFINE injection chamber.

The injection is performed with a sharp-edged stainless steel tube of $210 \mathrm{~mm}$ long, with a $4 \mathrm{~mm}$ outer diameter and a $2 \mathrm{~mm}$ inner diameter. The injector can be moved vertically over a $150 \mathrm{~mm}$ stroke by a motorized system located on the top of the chamber, to expand the field of view. The rig is fully instrumented with thermocouples, pressure gauges and mass flow meters as indicated in Fig. 2. The chamber is filled with Nitrogen and the pressure is controlled with a pressure regulator having a precision of $\pm 0.1 \mathrm{MPa}$. To warm up the ambient fluid, two MICA heating plates are placed at the bottom of the chamber and a heater (Fig. 2) is inserted just before the feeding of $\mathrm{N}_{2}$.

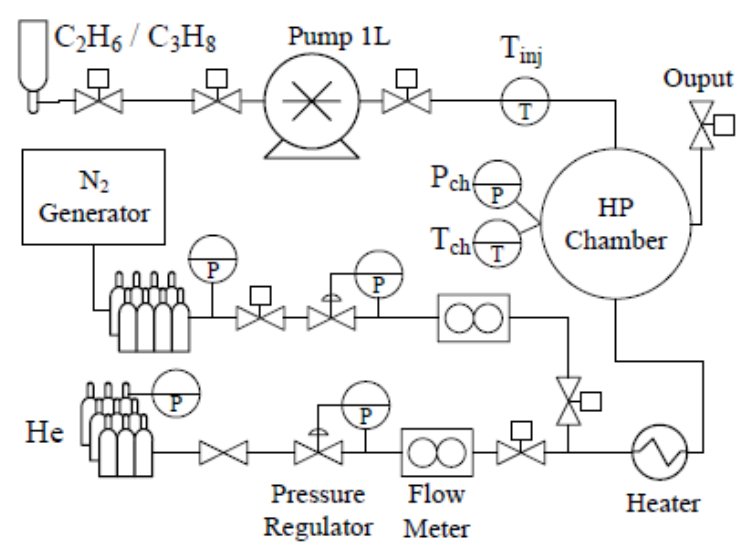

Figure 2. Flowchart of REFINE test bench.

Two optical diagnostics, diffused back-light illumination (DBI) and shadowgraphy, are used to quantify the injection and atomization of the working fluids. The main difference between these two techniques lies in the light source arrangement. For shadowgraphy, a collimated laser diode (Cavitar) is used whereas a stroboscope flashlamp (MVS2601) with a glass diffuser is used for DBI. Depending on the situation and on the desired information, different camera configurations were used, as indicated in Tab. 1. The DBI allows detecting the largest density gradients, which are essentially located at the liquid-gas interface contrary to shadowgraphy that detects smoother density gradient.

All the cases studied hereafter are summarized in Tab. 2, which provides the values of pressure $(P)$, temperature in the chamber $\left(T_{c h}\right)$ or upstream the injector $\left(T_{i n j}\right)$ and velocity $(V)$ calculated as the average of measurements made during the injection process. The \pm number indicates the variability of the measured quantity during this injection. The dimensionless numbers (Reynolds, Weber and Ohnesorge) are also provided. 
Table 1. Table of the different configurations of camera and light source with the associate designation code used in this manuscript.

\begin{tabular}{c|c|c|c|c}
\hline Code & Camera & Resolution $(\mathrm{px} / \mathrm{mm})$ & Field $(\mathrm{mm})$ & Light \\
\hline BG1 & BlueCougar-x24 & 27.0 & $59.3 \times 44.5$ & $\mathrm{DBI}$ \\
BG2-1 & BlueCougar-x25a & 53.5 & $45.8 \times 38.1$ & $\mathrm{DBI}$ \\
BG2-2 & BlueCougar-x25a & 130.5 & $18.8 \times 15.7$ & Shadowgraphy \\
PCO-1 & PCO Edge 5.5 & 58.0 & $44.1 \times 37.2$ & $\mathrm{DBI}$ \\
PCO-2 & PCO Edge 5.5 & 121.5 & $294.3 \times 17.8$ & $\mathrm{DBI}$
\end{tabular}

Table 2. Tests case conditions with dimensionless numbers: Reynolds $(R e)$, liquid and gaseous $\mathrm{Weber}_{(\mathrm{We}}$, We $\left.\mathrm{We}_{g}\right)$ and Ohnesorge $(O h)$.

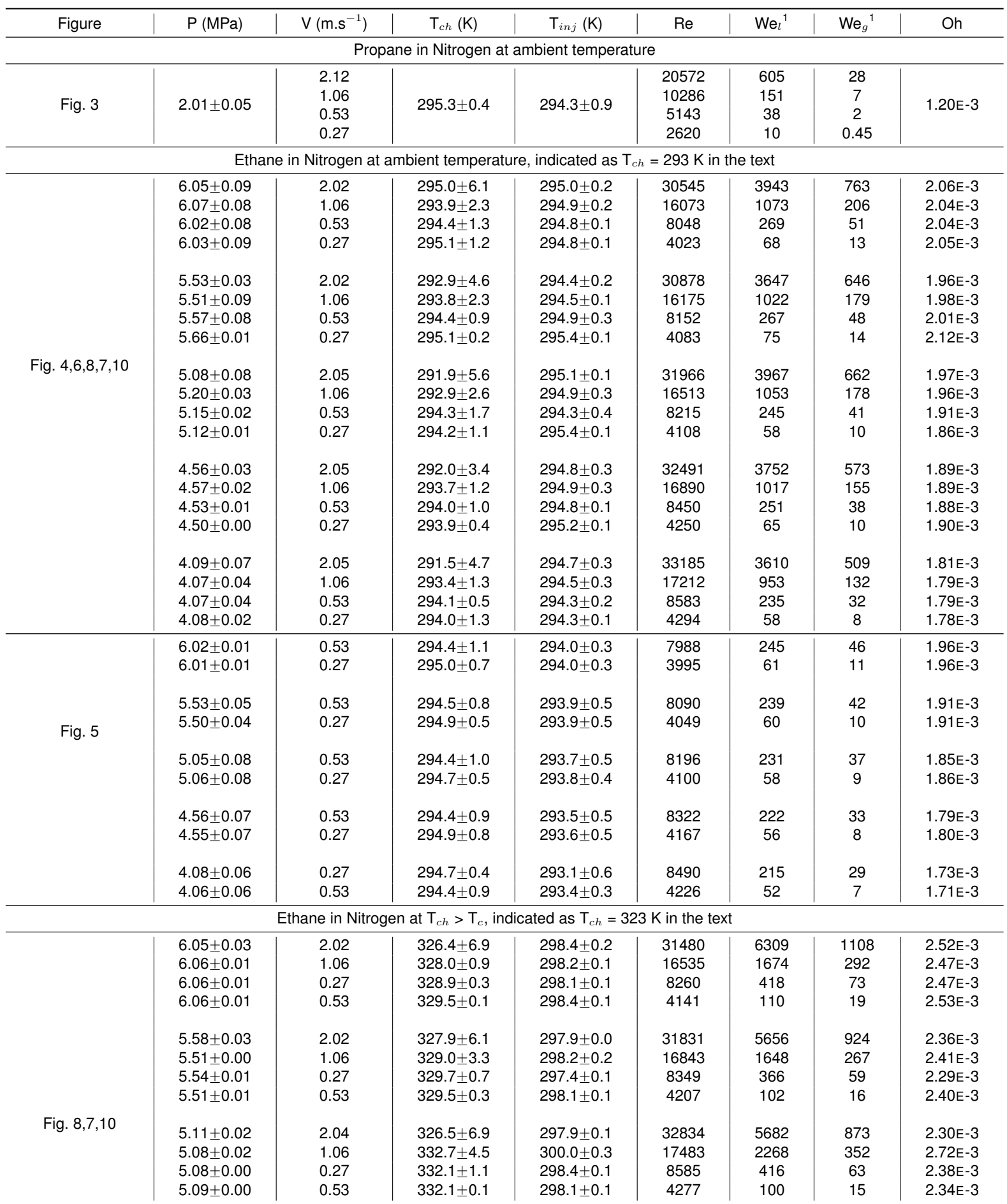

\footnotetext{
${ }^{1}$ Considering pure Ethane at $\mathrm{T}_{i n j}$ position and taken a surface tension from NIST (http://webbook.nist.gov/chemistry/) in liquid phase on the evaporation line at the $\mathrm{T}_{i n j}$
} 


\section{Results and discussion}

The behavior of a cylindrical jet discharging into a quiescent atmosphere is commonly characterized [15] by the jet stability curve as shown in Fig. 3. This curve shows the variation of the jet breakup length $\left(L_{b u}\right)$ as a function of the discharge velocity. The breakup length is defined as the distance between the injection orifice and the first discontinuity in the liquid jet. Five flow regimes are identified. They are respectively named the dripping regime $(a \rightarrow b)$, Rayleigh $(b \rightarrow c)$, first wind-induced $(c \rightarrow d)$, second wind-induced $(d \rightarrow e)$ and atomization regimes $(>e)$. The dripping regime is not of interest here.

Images of a Propane jet discharging into an atmosphere of nitrogen at $293 \mathrm{~K}$ and $2 \mathrm{MPa}$ are presented along the stability curve in Fig. 3. Refraction of light near the liquid-gas interface yields to steep grey level gradient making easier the localization of the interface. Brighter areas in the center are also due to the refraction of light which is less deviated when going through the jet axis. The more the surface is corrugated, the more deviated is the light making the jet darker. For $V=0.25 \mathrm{~m} . \mathrm{s}^{-1}$, the jet morphology is characteristic of the Rayleigh regime. Increasing the velocity up to $0.5 \mathrm{~m} . \mathrm{s}^{-1}$, the breakup length becomes greater than the field of view. The discharge velocity then approaches the critical velocity corresponding to point $c$. For $V=1 \mathrm{~m} \cdot \mathrm{s}^{-1}$ the breakup length becomes shorter, confirming that the critical velocity has been exceeded. The surface of the jet becomes corrugated and the first wind-induced regimes is reached. For $V=2 \mathrm{~m} . \mathrm{s}^{-1}$, disturbances on the jet surface are getting stronger and $L_{b u}$ is no longer observable within this field of view, i.e. the point $d$ is passed and the jet is in the second wind-induced regime.

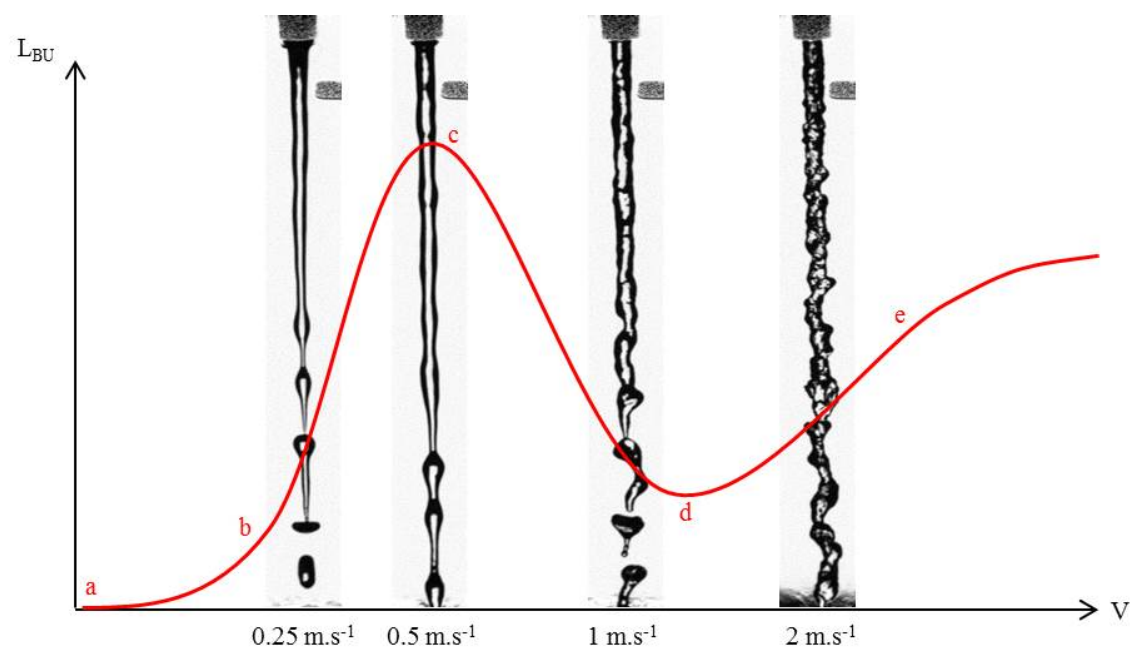

Figure 3. Injection of Propane at 2.0 MPa and ambient temperature along the stability curve [15](BG1).

Images of a jet of Ethane into Nitrogen at $293 \mathrm{~K}$ and 6.0 MPa are shown in Fig. 4. The breakup length of Ethane jet has been investigated for $V=0.25 \mathrm{~m} . \mathrm{s}^{-1}$ and $V=0.5 \mathrm{~m} . \mathrm{s}^{-1}$. For $V \geqslant 1 \mathrm{~m} . \mathrm{s}^{-1}$, the atomization starts only a few diameters downstream the injection orifice and the cloud of drops around the jet makes difficult the estimation of $L_{b u}$. The intact core length is thus commonly used in these conditions to characterize the jet atomization [16].

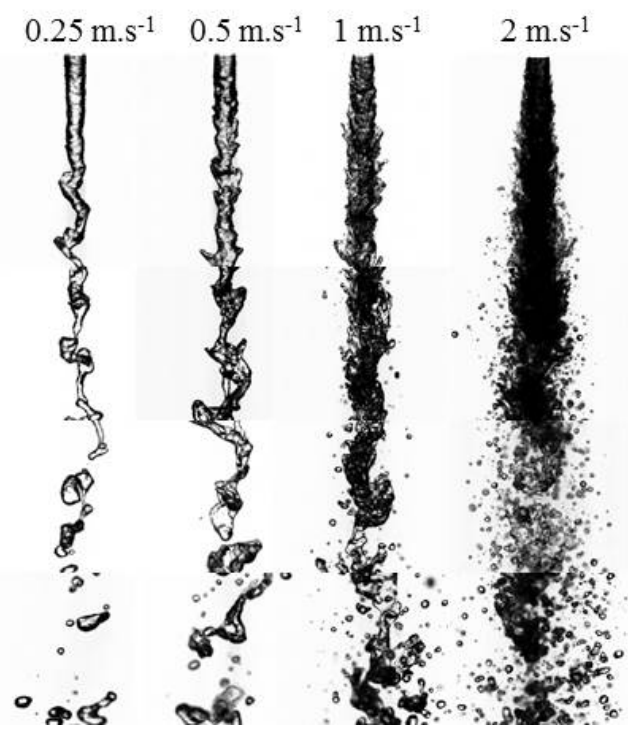

Figure 4. Injection of Ethane at 6.0 MPa and ambient temperature (PCO-2). 
Statistics on $L_{b u}$ of the Ethane jet was estimated for the two lowest discharge velocities. Since the jet deviates from its central axis, both perpendicular visual axes were used (BG2-1 \& PCO-1) to make sure that no drop or fluid deformations hide the first discontinuity of liquid. 600 two-level images were taken and analyzed for each case to compute the probability density functions (pdf) for $L_{b u}$ (see Fig. 5). For all the studied ambient pressures, the slowest velocity gives the shortest breakup length, meaning that $L_{b u}$ increases with the discharge velocity. Since the shape of the jet (see Fig. 4) has nothing in common with a Rayleigh jet, those jets can be classified in the second wind-induced regime. It can also be observed that the pdf of $L_{b u}$ are wider for a larger velocity and for $V=0.25 \mathrm{~m} . \mathrm{s}^{-1}$ the maximum peak is better defined. No conclusion could be drawn on the influence of pressure on the breakup length.
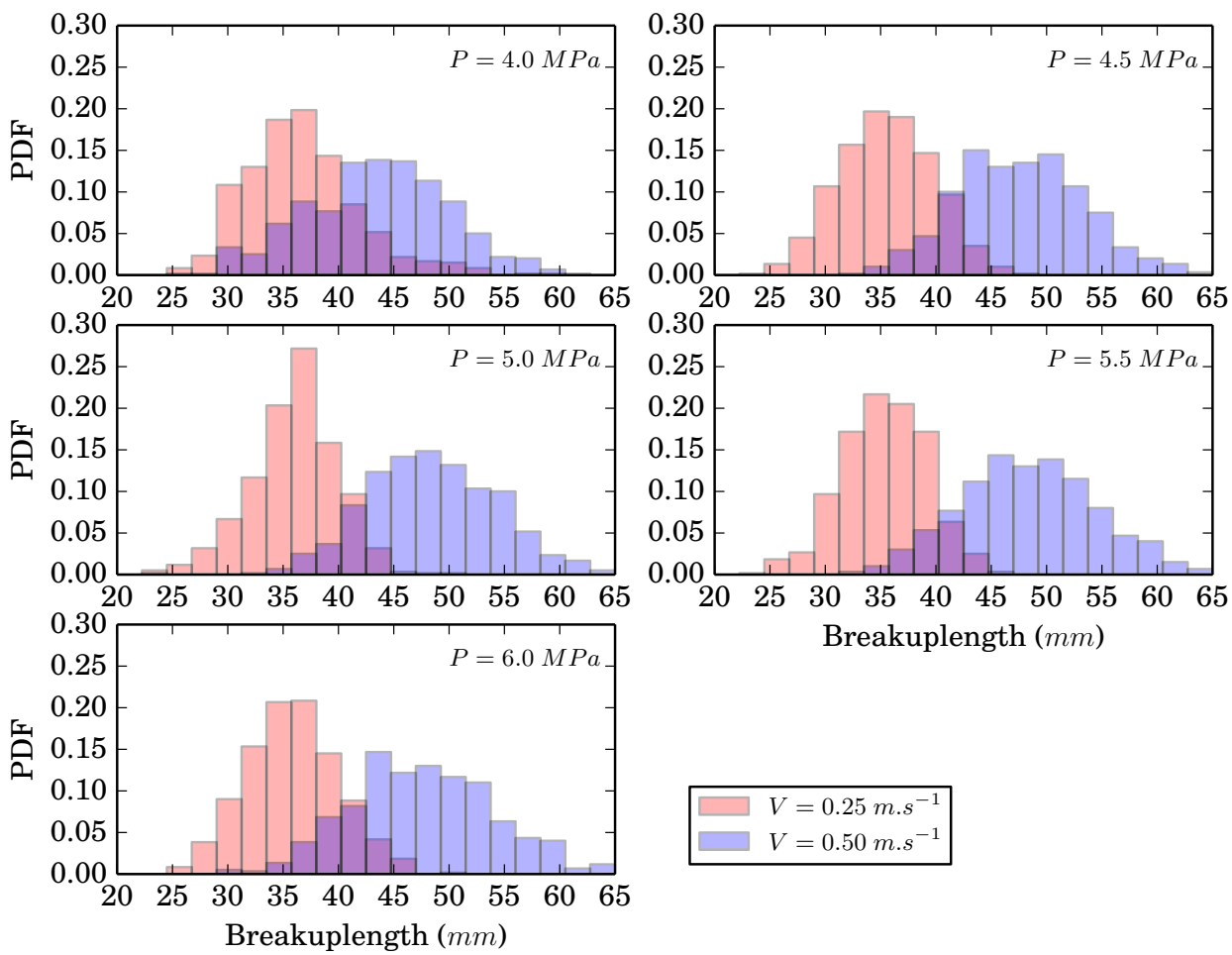

Figure 5. PDF of 600 breakup lengths of Ethane jet at $293 \mathrm{~K}$. Pressure from 4.0 MPa to 6.0 MPa.

The impact of the chamber pressure on jet disintegration is illustrated in Fig. 6 for Ethane injected into $\mathrm{N}_{2}$ at $293 \mathrm{~K}$ with a fixed discharge velocity of $1 \mathrm{~m} . \mathrm{s}^{-1}$. The pressure ranges from 4.0 MPa to 6.0 MPa, i.e. from subcritical to supercritical pressure if referring to Ethane critical pressure. Increasing the pressure leads to more perturbed jets with smaller drops, similarly to the observations done by Oschwald [17] for an injection of $\mathrm{N}_{2}$ at $5 \mathrm{~m} . \mathrm{s}^{-1}$ into $\mathrm{N}_{2}$ at rest.

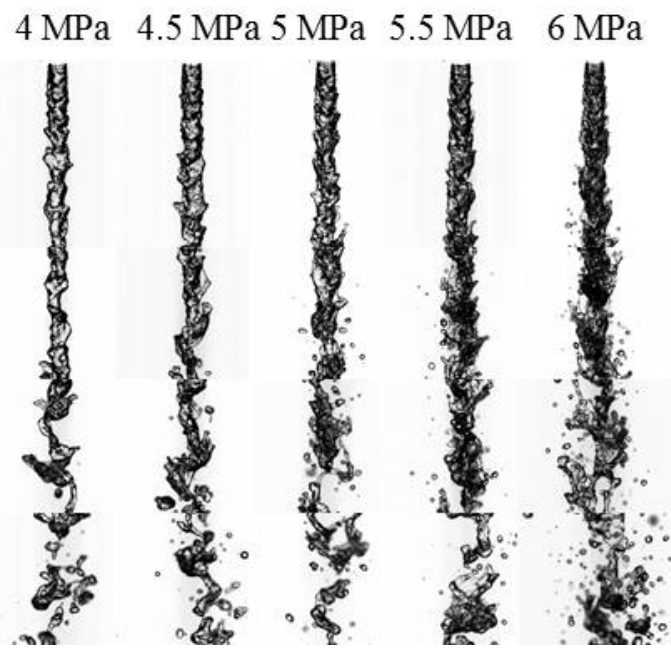

Figure 6. Injection of Ethane into Nitrogen at $V=1 \mathrm{~m} \cdot \mathrm{s}^{-1}$ for different levels of ambient pressure with $T=293 \mathrm{~K} .4$ images are used to create a field of visualization of $66.1 \mathrm{~mm}$ length (PCO-2). 
Table 3. Measured temperatures $T_{c h}, T_{i n j}$ and $T_{j e t}$ in the case of an injection of Ethane into $\mathrm{N}_{2}$ for different velocities.

\begin{tabular}{c|c|c|c|c|c}
\hline$P(\mathrm{MPa})$ & $V\left({\left.\mathrm{~m} . \mathrm{s}^{-1}\right)}\right.$ & $T_{c h}(\mathrm{~K})$ & $T_{i n j}(\mathrm{~K})$ & $T_{j e t}(\mathrm{~K})$ at $6 \mathrm{~mm}$ & State $^{2}$ \\
\hline 6.0 & 0.25 & 327 & 298 & 308 & Supercritical \\
6.0 & 0.50 & 325 & 297 & 303 & Liquid \\
6.0 & 1.00 & 323 & 298 & 300 & Liquid \\
6.0 & 2.00 & 328 & 298 & 298 & Liquid
\end{tabular}

To explore the effect of the ambient temperature, various configurations of Ethane jets into Nitrogen for four discharge velocities and a temperature of $\mathrm{N}_{2}$ set to $T=293 \mathrm{~K}<T_{c}$ or $T=323 \mathrm{~K}>T_{c}$ are shown in Fig. 7. Pressure is fixed to $6.0 \mathrm{MPa}$, i.e. above the critical pressure of Ethane. The temperature of the jet $\left(T_{j e t}\right)$ measured in the fluid at the nozzle exit with an exposed junction thermocouple placed $6 \mathrm{~mm}$ downstream from the nozzle exit are summarized in Tab. 3. These temperature measurements were done on different experiments, i.e. not simultaneously to image acquisition. This temperature, $T_{j e t}$, decreases with the increase of the velocity. The discharge velocity must be considered as a key-point parameter since it induces modification in the ratio between characteristic convection and thermal times

For the lowest injection velocities $\left(V=0.25 \mathrm{~m} . \mathrm{s}^{-1}\right.$ and $\left.V=0.5 \mathrm{~m} . \mathrm{s}^{-1}\right)$, the temperature has a clear impact on jet disintegration since $T_{j e t}$ is close to the critical temperature of Ethane ( $T_{c}=305 \mathrm{~K}$ ). For example, a diffuse jet with dense pockets appears for $T_{c h}=323 \mathrm{~K}$ and $V \leqslant 0.5 \mathrm{~m} . \mathrm{s}^{-1}$ whereas a liquid-gas interface is clearly visible for $T_{c h}=293 \mathrm{~K}$.

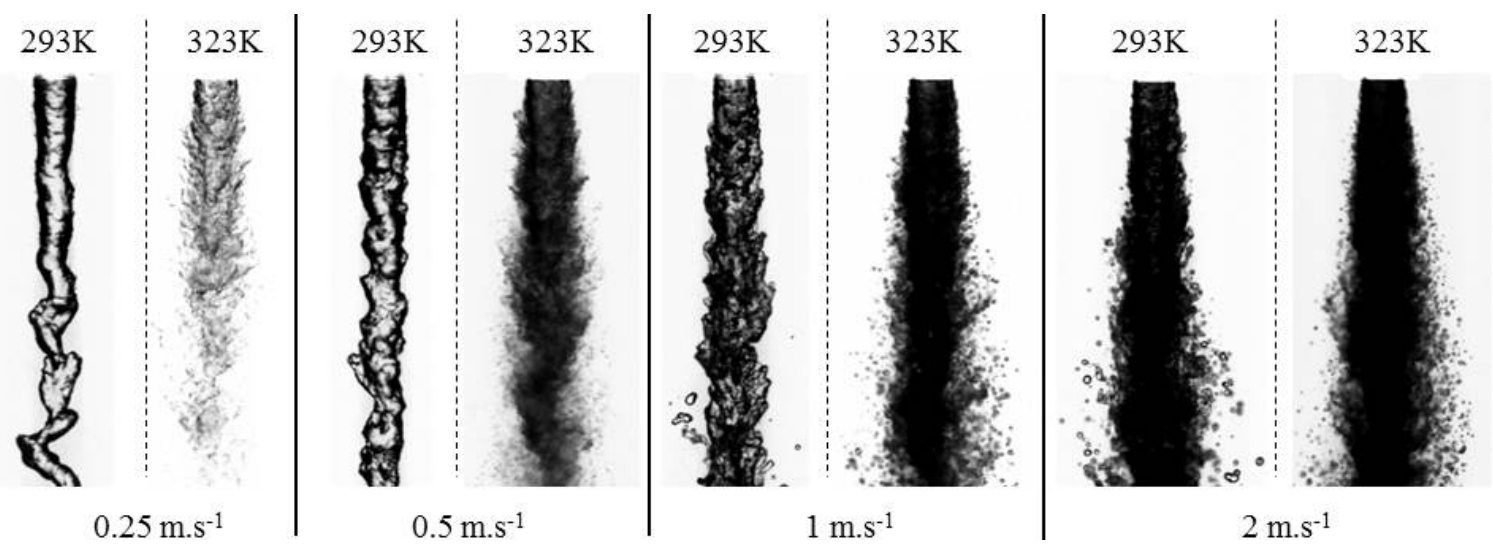

Figure 7. Jets of Ethane in Nitrogen at $6 \mathrm{MPa}(\mathrm{PCO} 2)$.

Increasing the velocity to $V=1 \mathrm{~m} . \mathrm{s}^{-1}$, small fluid elements identified as drops appear whatever $T_{c h}$. However, there are more droplets identified for $T=323 \mathrm{~K}$. The same observation is done for $V=2 \mathrm{~m} . \mathrm{s}^{-1}$. Hence, having a temperature of the ambient fluid above the critical temperature of the injected fluid strongly modifies the jet behavior. The interaction between the injected fluid and the ambient one is greater for low injection velocities where a diffusion process is observed, but modifies also the atomization process at higher injection velocity as stated in [3, 18].

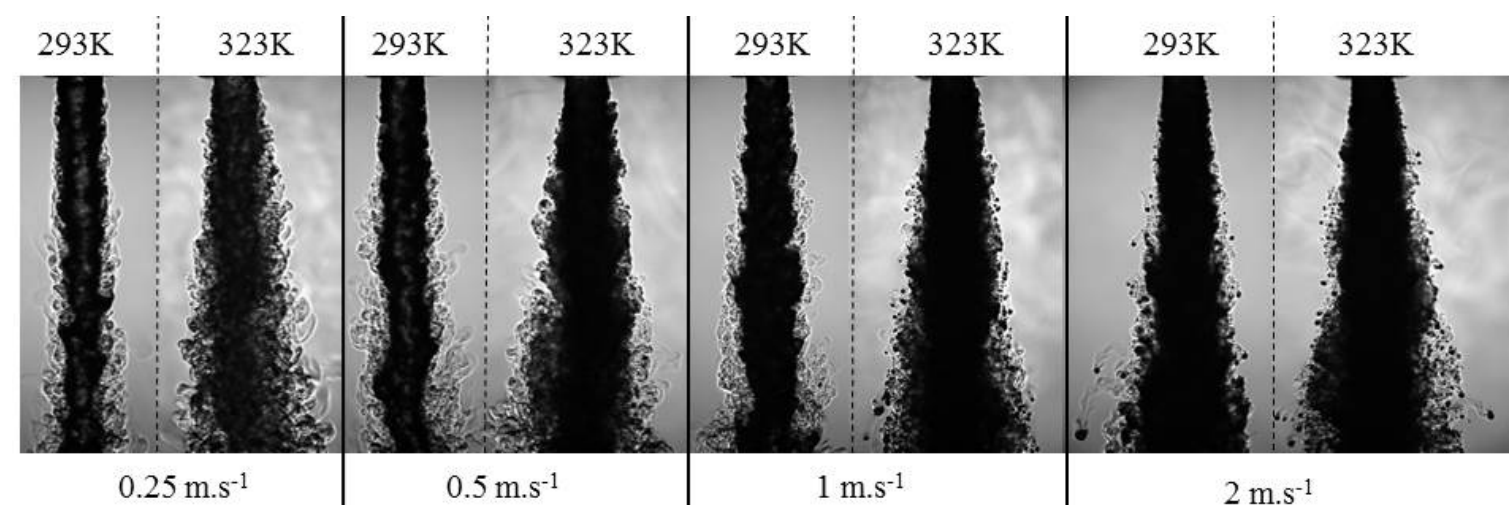

Figure 8. Jets of Ethane in Nitrogen at $6 \mathrm{MPa}$ (BG2-2).

With DBI visualizations some finger-like structures are observed at the jet interface. In order to analyze those structures, shawdowgraph visualizations were achieved to explore the density gradient around the liquid jet (or around the dense core). Accordingly, shadowgraph images (Fig. 8) have been taken simultaneously to DBI images ( Fig. 7) on the perpendicular axis. A grey level gradient clearly appears around the liquid. It consists of a $\mathrm{C}_{2} \mathrm{H}_{6}-\mathrm{N}_{2}$

\footnotetext{
${ }^{2}$ Considering pure Ethane at the $T_{\text {jet }}$ position.
} 
mixing layer varying radially from pure Ethane in the center of the jet to pure Nitrogen far from the jet.

Two topologic parameters are then defined from shadowgraph images: the dark-core angle and the spreading angle also named growth rate [19]. From the normalized images of raw shadowgraphs, a two-level image is created. The black and white delimitation gives an interface between dense and light fluid. The calculation of the standard deviation of 120 two-level shadowgraph images yields the probability of presence of this interface on a grey-level scale. Only the values above $90 \%$ of the maximum of this probability are retained to define the area delimited by the dark-core angle $\theta_{d}$ (inside boundary in red on Fig. 9) and the spreading angle $\theta_{s}$ (outside boundary in green). The spreading angle for different pressures, velocities and chamber temperatures of an Ethane jet are plotted in Fig. 10.
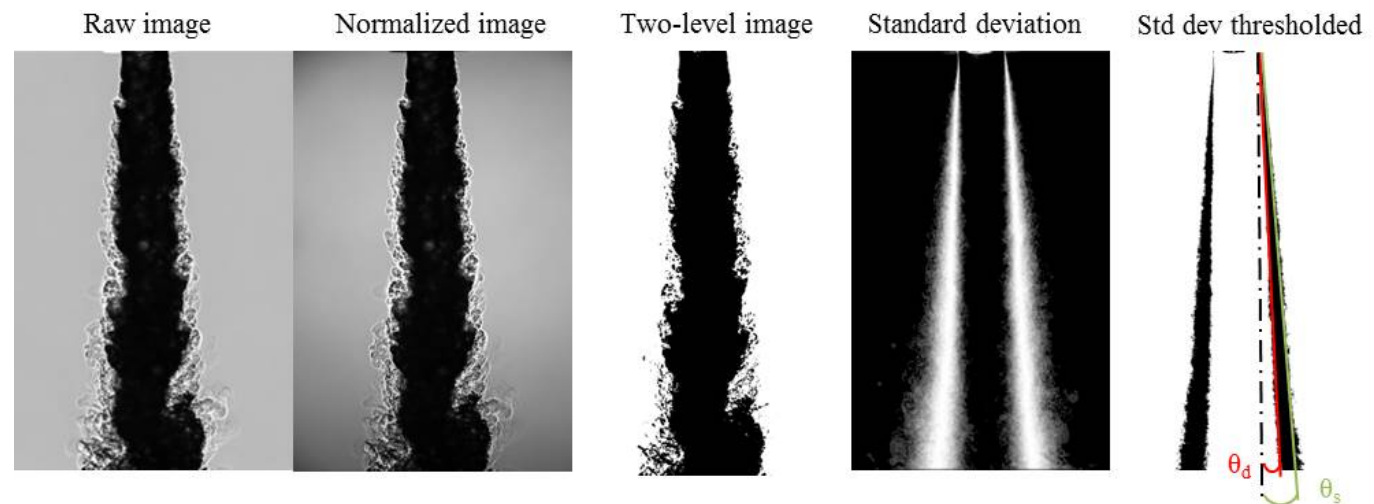

Figure 9. Image processing from raw image to the threshold of the standard deviation. On the last image: the dark-core angle $\theta_{d}$ is on the inside boundary in red and the spreading angle $\theta_{s}$ is outside in yellow.

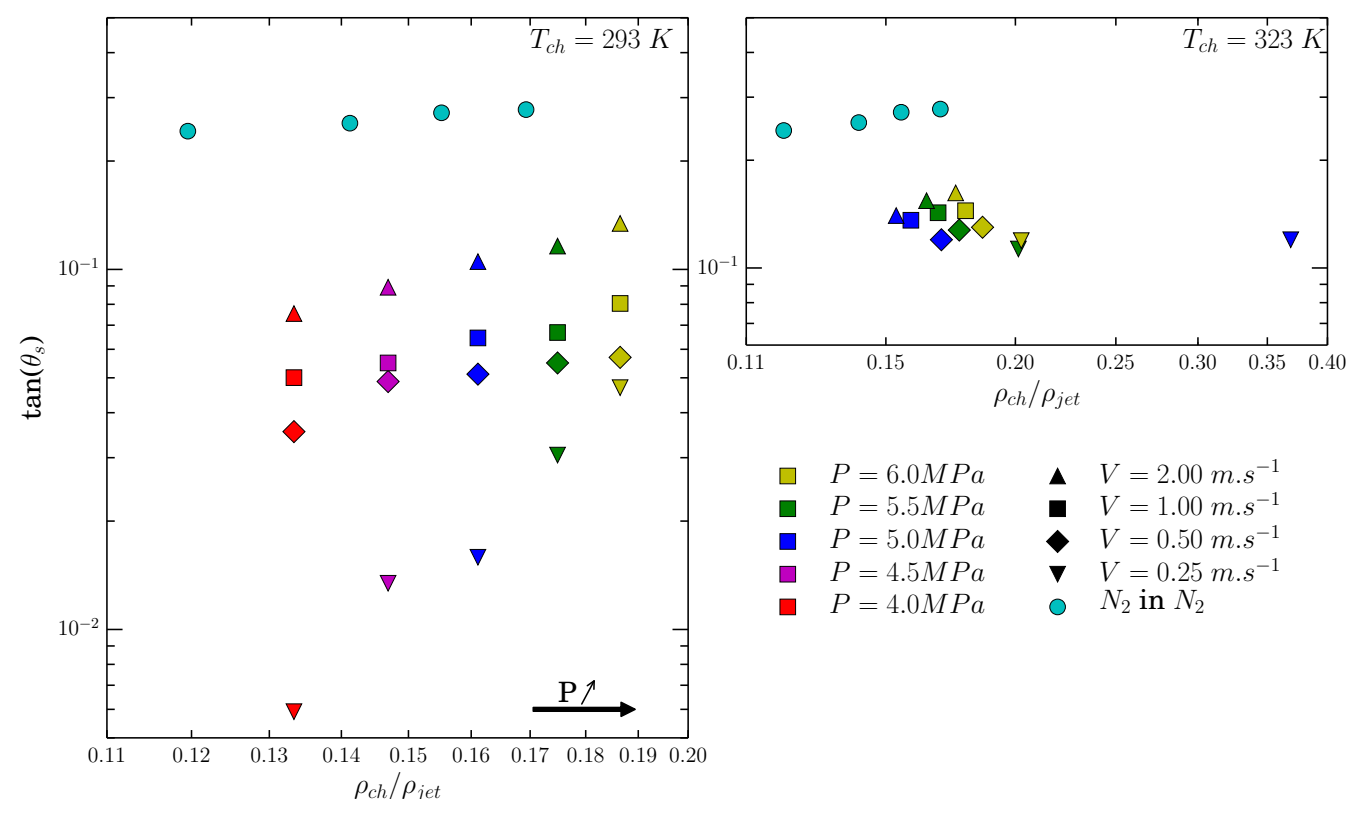

Figure 10. Spreading angle versus the chamber to injectant density ratio. For comparison, spreading angle of liquid $\mathrm{N}_{2}$ injection in $\mathrm{N}_{2}$ at ambient temperature from Fig. 8 of Chehroudi's paper [12].

To be compared to the results summarized by Chehroudi in [12], the spreading angle is plotted against the ambient over injectant density ratio. The density of pure fluids is based on the measured temperature (Tab. 3) and estimated from NIST tables. For example, if $T_{c h}=323 \mathrm{~K}$, then the temperature of Ethane considered for NIST tables is $T_{j e t}$. For each velocity, the growth rate increases with the pressure (also with the density ratio), which is in agreement with the other studies. At a given ambient pressure, the spreading angle increases with the velocity. Ethane spreading angles are smaller to the AFRL data for liquid $N_{2}$ in $N_{2}$ [12] having similar density ratios. This difference can be due to the velocity range in our experiments, which is one of the lowest in the literature but no indication of velocity is given on the original plot of Chehroudi (Fig. 8 in [12]). Increasing the temperature makes a significant change of flow topology at low velocities. In Fig. 10 (right), the injection of Ethane at $V=0.25 \mathrm{~m} . \mathrm{s}^{-1}$ under a pressure of $5.0 \mathrm{MPa}$ exhibits a density ratio of $\rho_{c h} / \rho_{\text {jet }}=0.35$ contrary to configurations at higher pressure $\left(\rho_{c h} / \rho_{j e t} \approx 0.20\right)$. Indeed decreasing the pressure makes the working conditions closer to the critical point. The limit of plotting the spreading angle against the density ratio is that when the chamber is heated, the jet temperature varies along the jet. There is even a temperature gradient in Nitrogen evidenced by the non-uniform grey level in shadowgraph images far from the jet at $323 \mathrm{~K}$ in Fig. 8. 


\section{Conclusions}

A new fully instrumented experimental test-bench has been developed at CORIA lab to study the injection of inert fluids under supercritical conditions. In this study, Ethane and Propane are injected into a cold or warm environment of Nitrogen. The knowledge of the behavior of those fluids has been detailed. Results for the breakup length and the spreading angle are in agreement with the literature. It has been shown that the temperature of the ambient fluid played a major role in jet disintegration when pressure exceeds the critical pressure of the injected fluid. In case of an ambient temperature higher than the critical temperature of injected fluids a diffuse mixing process occurs for low discharge velocities. For a higher injection velocities, a process of atomization appears but with a larger spatial distribution of drops. These results were obtained by shadowgraphy and diffused backlight illumination that gave complementary information. Other optical diagnostics as schlieren and CBOS will be soon implemented to complement these first results. A X-ray analysis is currently under progress to deliver a density gradient measurement and to give additional characterization of the jet behaviors.

\section{Acknowledgements}

This work was funded by the ANR (Agence Nationale de la Recherche) research project REFINE (Real-gas Effects on Fluid Injection: a Numerical and Experimental study), Grant No. ANR-13-BS09-0007.

\section{References}

[1] Dahms, R. N., and Oefelein, J. C., 2013. "On the transition between two-phase and single-phase interface dynamics in multicomponent fluids at supercritical pressures". Physics of Fluids (1994-present), 25(9), p. 092103.

[2] Dahms, R. N., and Oefelein, J. C., 2015. "Liquid jet breakup regimes at supercritical pressures". Combustion and Flame, 162(10), pp. 3648-3657.

[3] Oschwald, M., Smith, J. J., Branam, R., Hussong, J., and Schik, A., 2006. "Injection of fluids into supercritical environments". Combust. Sci. and Tech., 178, pp. 49-100.

[4] Petit, X., Ribert, G., Lartigue, G., and Domingo, P., 2013. "Large-eddy simulation of supercritical fluid injection". The Journal of Supercritical Fluids, 84, pp. 61-73.

[5] Petit, X., Ribert, G., and Domingo, P., 2015. "Framework for real-gas compressible reacting flows with tabulated thermochemistry". The Journal of Supercritical Fluids, 101, pp. 1-16.

[6] Ribert, G., Petit, X., and Domingo, P., 2016. "High-pressure methane-oxygen flames. analysis of sub-grid scale contributions in filtered equations of state". The Journal of Supercritical Fluids, 121, pp. 78-88.

[7] Turns, S. R., 2006. Thermodynamics: concepts and applications. Cambridge University Press.

[8] Manin, J., Bardi, M., Pickett, L., Dahms, R., and Oefelein, J., 2014. "Microscopic investigation of the atomization and mixing processes of diesel sprays injected into high pressure and temperature environments". Fuel, 134, pp. 531-543.

[9] Segal, C., and Polikhov, S., 2008. "Subcritical to supercritical mixing". Phys. Fluids, 20, p. 052101.

[10] Mayer, W., Schik, A. H., Vielle, B., Chauveau, C., Gškalp, I., Talley, D. G., and Woodward, R. D., 1998. "Atomization and breakup of cryogenic propellants under high-pressure subcritical and supercritical conditions". Journal of Propulsion and Power, 14(5), pp. 835-842.

[11] Chehroudi, B., Talley, D., and Cohn, R., 2002. "Visual characteristics and initial growth rates of round cryogenic jets at subcritical and supercritical pressures". Physics of Fluids, 14-2, pp. 850-861.

[12] Chehroudi, B., Talley, D., Mayer, W., Branam, R., and Smith, J., 2003. Understanding injection into high pressure supercritical environments. Tech. rep., DTIC Document.

[13] Mayer, W., Telaar, J., Branam, R., Schneider, G., and Hussong, J., 2003. "Raman measurements of cryogenic injection at supercritical pressure". Heat and Mass Transfer, 39, pp. 709-719.

[14] Manin, J., Bardi, M., Pickett, L., and Payri, R., 2016. "Boundary condition and fuel composition effects on injection processes of high-pressure sprays at the microscopic level". International Journal of Multiphase Flow, 83, pp. 267-278.

[15] Dumouchel, C., 2008. "On the experimental investigation on primary atomization of liquid streams". Exp. Fluids, 45, pp. 371-422.

[16] Charalampous, G., Hadjiyiannis, C., and Hardalupas, Y., 2016. "Comparative measurement of the breakup length of liquid jets in airblast atomisers using optical connectivity, electrical connectivity and shadowgraphy". Measurement, 89, pp. 288-299.

[17] Oschwald, M., Schneider, G., and Clauss, W., 2005. "Application of visualization techniques and quantitative optical diagnostics for the investigation of supercritical jet atomization". In The 8th International Symposium on Fluid Control, Measurement and Visualization.

[18] Chehroudi, B., 2012. "Recent experimental efforts on high-pressure supercritical injection for liquid rockets and their implications". International Journal of Aerospace Engineering, 2012.

[19] Chehroudi, B., Cohn, R., and Talley, D., 2002. "Cryogenic shear layers: experiments and phenomenological modeling of the initial growth rate under subcritical and supercritical conditions". Int. J. of Heat Fluid Flow, 23, pp. 554-563. 